\title{
DNA - Gold Nanoparticles Designed for mRNA Sensing in Cells: Imaging of the Gold Nanoparticles Using Two Photon Photoluminescence Spectroscopy
}

\author{
Maria-Eleni Kyriazi ${ }^{\mathrm{a}}$, Otto L. Muskens, ${ }^{\mathrm{a}, \mathrm{b}}$ and Antonios G. Kanaras ${ }^{\mathrm{a}, \mathrm{b} *}$

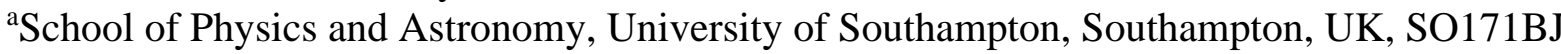 \\ ${ }^{\mathrm{b}}$ Institute for Life Sciences, University of Southampton, Southampton, UK, SO171BJ
}

Email: a.kanaras@ soton.ac.uk, phone: 00442380592466, fax: (+44) (0) 2380593910

\begin{abstract}
Recently DNA - coated gold nanoparticles have emerged as ideal tools for the detection of mRNA in cells using dye modified oligonucleotides. However, the tracking of the gold core has been hindered by the small size of the particle core. In this work we utilize a home built set up and $43 \mathrm{~nm}$ DNA - coated spherical gold nanoparticles for the simultaneous imaging of mRNA detection using fluorescence microscopy and the gold nanoparticle core using two photon photoluminescence (TPPL).
\end{abstract}

Keywords: DNA, mRNA, nanoparticles, colloids, drugs, cancer, TPPL

\section{INTRODUCTION}

In recent years the vast capabilities of DNA - coated gold nanoparticles (AuNPs) have been explored including their use as sensors and as therapeutic agents for the targeted treatment of diseases and/or skin conditions. ${ }^{1,2}$ In particular spherical AuNPs modified with a monolayer of synthetic oligonucleotides have been shown to accurately detect specific mRNA targets in live cells. In this design, AuNPs are coated with a thiol modified sense strand that is conjugated to the AuNP surface. To the sense, a shorter fluorophore modified oligonucleotide is hybridized termed flare strand. The sense sequence can be designed to detect a specific mRNA target thus when the target mRNA binds, the flare strand will be released due to competitive hybridization and a fluorescence signal will be detected. In the absence of the target the flare will remain bound and the fluorescence signal corresponding to the dye will be quenched by the gold core as shown in Scheme 1. ${ }^{3-5}$ Since their initial development, this design has not only been shown to be capable of the targeted detection of biomolecular targets such as mRNA and microRNA but has also been developed into a successful drug delivery agent. By incorporating intercalating drugs into the DNA duplex, it was demonstrated the targeted release only to cells expressing a specific mRNA target leading to efficient cell death. ${ }^{4-13}$ However, the intracellular fate of DNA - coated AuNP probes following cellular uptake is still under investigation. In order to assess nanoparticle intracellular fate, imaging of the gold core in conjunction with flare release would be of substantial interest. ${ }^{14}$

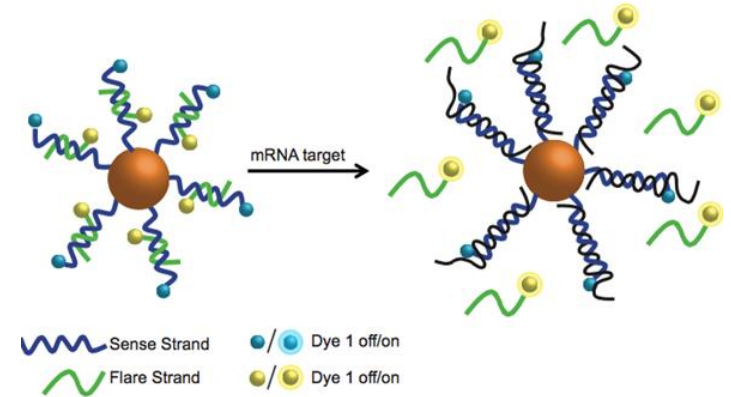

Scheme 1. Schematic illustration of nanoparticles used for live cell mRNA detection. Gold nanoparticles are conjugated to an oligonucleotide strand (sense strand-blue color). A second shorter oligonucleotide strand is hybridized on the sense strand (flare-strand-green). Dyes are conjugated to both of the oligo strands. 
Apart from acting as a scaffold for DNA attachment, the gold nanoparticle core can also demonstrate interesting optical properties that can be used for its direct imaging within cells. AuNPs that display strong TPPL are attractive as contrast agents for bioimaging as it allows for non-invasive imaging of living tissue with high penetration depths, 3D mapping capabilities and reduced photobleaching. ${ }^{5}$ Furthermore, TPPL strongly increases the signal to noise ratio and is appealing due to the absence of a background signal, which is important when imaging in scattering media. For prolonged live cell tracking, quantum dots are usually utilized as a feasible option due to their enhanced stability. However, they are composed of heavy metals that are cytotoxic making them unsuitable for in vivo clinical applications. ${ }^{5,15,16}$ AuNPs on the other hand, are biocompatible and have a large two-photon action cross-section making them ideal alternative candidates. $^{5}$

Research by Gao et al. suggested that TPPL is shape and size dependent with branched AuNPs and AuNRs displaying the strongest TPPL. ${ }^{5}$ AuNRs as small as $8 \times 40 \mathrm{~nm}$ can be easily detected with a high signal to noise ratio. Furthermore, they are easily functionalized, they are non-cytotoxic and their longitudinal plasmon resonance can be tuned to the near infrared wavelengths where biological tissue exhibits relatively small extinction coefficients. ${ }^{16}$ For example, a recent study involved the use of folate nanorods for the targeted delivery to KB cells (a tumor cell line derived from oral epithelium), which is known to overexpress folate receptors. Via TPPL imaging it was noticed that $6 \mathrm{~h}$ after incubation a large number of nanoparticles was observed on the outer membrane with complete internalization occurring after $17 \mathrm{~h}$. Furthermore, when nanorods were incubated with a cell line lacking these receptors little to no uptake was observed. ${ }^{17}$ However, TPPL imaging has also proven useful for 3D imaging using cancer cells supported in a collagen matrix to mimic tissue. Durr et al. also showed that by conjugating anti-EGFR antibodies to AuNRs of $14 \mathrm{~nm}$ in width and $48 \mathrm{~nm}$ in length, EGFR overexpressing A431 skin cancer cells could be imaged by TPPL with characteristic bright rings being observed as well as bright spots in the cytoplasm, which they suggested that it was indicative of endosomal uptake. ${ }^{15}$ Furthermore, Wang et al. successfully demonstrated the TPPL imaging of nanorods in vivo. By monitoring the TPPL signal the flow of AuNRs through mouse ear blood vessels could be tracked. TPPL signal was found to be 3 times higher than the background and interestingly no signal could be detected after 30 minutes indicating successful clearance from circulation. ${ }^{18}$ On the other hand, Liu et al. demonstrated how branched nanoparticles could also be imaged in vivo. Branched AuNPs have a high TPPL signal mainly due to their sharp spikes that result in larger TPPL action crosssection. Their study highlighted the ability of successfully imaging branched nanoparticles of two different sizes (30 and $60 \mathrm{~nm}$ ) after intravenous injection into mice with xenograft sarcomas. However, when testing $12 \mathrm{~nm}$ AuNPs no TPPL signal could be detected. ${ }^{19}$ In contrary to this result, Rane et al. found that TPPL imaging of AuNPs ranging from 10 to $70 \mathrm{~nm}$ coated with an oligonucleotide shell could be successfully utilized to image in 2D and 3D models of the HCT116 cell line, a colorectal carcinoma cell line. ${ }^{20}$

In this paper, we demonstrate the simultaneous detection of mRNA using fluorescence and the gold core by using TPPL in cells. For these experiments, a home built setup was designed capable of performing both TPPL and fluorescence microscopy.

\section{MATERIALS AND METHODS}

\subsection{Materials}

Sodium tetrachloroaurate (III) dihydrate, bis(p-sulfonatophenyl)phenyl phosphine dihydrate dipotassium salt (BSPP), trisodium citrate and phosphate buffered saline (PBS) were purchased from Sigma Aldrich. Minimum essential media (MEM), Pen/Strep, L glutamine, trypsin, nystatin, fetal bovine serum (FBS), and HBSS for cell culturing were purchased from Invitrogen. Oligonucleotides, including their modifications, were obtained from ATD Bio.

\subsection{Synthesis of $43 \pm 4 \mathbf{n m}$ spherical AuNPs}

For the synthesis of larger particles (> $20 \mathrm{~nm}$ ) a kinetically controlled seeded growth strategy was followed developed by Puntes et al. ${ }^{21}$ This method allows for the synthesis of monodisperse citrate-stabilized AuNPs with a quasi-spherical shape and a narrow size distribution. In detail, the Au seed solution was firstly prepared by heating a solution of sodium citrate in Milli-Q water to boiling conditions $(150 \mathrm{~mL}, 2.2 \mathrm{mM})$ under vigorous stirring. A three-necked round-bottomed flask $(250 \mathrm{~mL})$ was used, which was fitted with a condenser to avoid solvent evaporation. Once boiling had commenced, a solution of $\mathrm{NaAuCl}_{4}(25 \mathrm{mM}, 1 \mathrm{~mL})$ was quickly injected. After boiling for a further 10 min the color of the solution had changed from yellow to bluish grey to light pink. Immediately after, the temperature of the solution was cooled to 90 ${ }^{\circ} \mathrm{C}$ and a $\mathrm{NaAuCl}_{4}$ solution $(1 \mathrm{~mL}, 25 \mathrm{mM})$ was injected. After $30 \mathrm{~min}$ one more addition of a gold solution $(1 \mathrm{~mL}, 25$ $\mathrm{mM}$ ) was performed to form the initial generation of particles $(\mathrm{g} 0)$. Then, the sample was diluted by extracting $55 \mathrm{~mL}$ of sample and adding $53 \mathrm{~mL}$ of Milli-Q water and sodium citrate solution $(2 \mathrm{~mL}, 60 \mathrm{mM})$. This solution was used as a seed 
solution, which was injected with three consecutive gold solution additions every 30 min at $90{ }^{\circ} \mathrm{C}$ to generate the next generation of particles (g1). After synthesis was complete, citrate-capped gold nanospheres were purified by two rounds of centrifugation $(6,000 \mathrm{rpm}, 20 \mathrm{~min})$, decantation and re-dispersion in Milli-Q water. Then, larger aggregates were removed by filtration $\left(0.2 \mu \mathrm{m}\right.$, VWR). Sample was stored at $4{ }^{\circ} \mathrm{C}$ prior to further functionalization

\subsection{Functionalization of $43 \pm 4 \mathrm{~nm}$ spherical AuNPs with synthetic oligonucleotides}

Oligonucleotide attachment to larger spherical AuNPs was successfully achieved using a pH-assisted protocol. This method allows for the instantaneous DNA adsorption onto the gold surface via the use of a pH 3 citrate buffer. ${ }^{22}$ Briefly citrate capped $43 \pm 4 \mathrm{~nm}$ spherical AuNPs $(1.35 \mathrm{~mL}, 10 \mathrm{pmol})$ were mixed with a $3000 \mathrm{x}$ excess of thiol terminated oligonucleotides $(200.88 \mu \mathrm{L}, 30 \mathrm{nmol})$ and vigorously stirred for $1 \mathrm{~min}$. This was followed by the addition of a $\mathrm{pH} 3$ citrate buffer $(30 \mu \mathrm{L}, 500 \mathrm{mM})$. After $3 \mathrm{~min}$, the $\mathrm{Na}^{+}$ion concentration was increased via the addition of a $\mathrm{NaCl}$ solution $(225 \mu \mathrm{L}, 2 \mathrm{M})$ to reach a final concentration of $0.3 \mathrm{M}$ within the final solution. The solution was left to stir for $1 \mathrm{~min}$ before three rounds of centrifugation $(12,000 \mathrm{rpm}, 15 \mathrm{~min})$, decantation and redispersion in PBS. The purified solution was stored at $4{ }^{\circ} \mathrm{C}$ prior to their use in further applications. For flare hybridization, the oligonucleotide flare strands $(150.2 \mu \mathrm{L}, 15 \mathrm{nmol})$ were incubated with purified spherical AuNPs. The solution was heated to $60{ }^{\circ} \mathrm{C}$ and allowed to slowly cool to room temperature followed by purification via three rounds of centrifugation $(16,400 \mathrm{rpm}, 20 \mathrm{~min})$ and redispersion in PBS. Solution was stored at $4{ }^{\circ} \mathrm{C}$.

\subsection{Incubation of DNA - coated AuNPs with cells}

Human fetal lung fibroblast (MRC 5) cells were grown on a coverslip in MEM until $90 \%$ confluent. Once this confluency was reached, media was removed and exchanged with fresh media containing DNA - coated AuNPs $(55 \mu \mathrm{L}$, $2 \mathrm{pmol})$. Cells were then incubated with the solution at $37^{\circ} \mathrm{C}$ for $18 \mathrm{~h}$.

\subsection{Cell fixation}

Media was removed from confluent cells, which were washed with PBS $(3 \times, 5 \mathrm{~min})$. After washing, cells were rinsed with $4 \%$ paraformaldehyde (PFA) in piperazine-N,N'-bis(2-ethanesulfonic acid) (PIPES) and left for $1 \mathrm{~h}$ at $37{ }^{\circ} \mathrm{C}$. Cells were then washed with PBS $(3 \times, 5 \mathrm{~min})$ followed by an incubation step with 4',6-diamidino-2-phenylindole (DAPI) (1/500) in PBS for 15 min. Coverslips were then mounted with Mowiol Glycerol onto glass slides.

\section{RESULTS AND DISCUSSION}

\subsection{Synthesis of $43 \pm 4 \mathbf{n m}$ spherical AuNPs}

In order to accommodate both size and dispersity requirements, larger AuNPs were synthesized according to a protocol developed by the Puntes group (see section 2.2). ${ }^{21}$ This synthesis involves the use of spherical AuNPs as seeds, which are gradually grown by further additions of both the gold precursor and citrate. After every three additions half the sample was extracted (termed generation $\mathrm{n}$ ) for further analysis whereas the other half was further grown into larger size AuNPs. In this case AuNPs of generation 1 (G1) were synthesized ( $40 \mathrm{~nm}$ in size), analyzed and used in further applications. Citrate ligands were replaced with BSPP to increase AuNP stability and allow for concentration via centrifugation. A representative TEM image and size distribution analysis is presented in Figure 1.
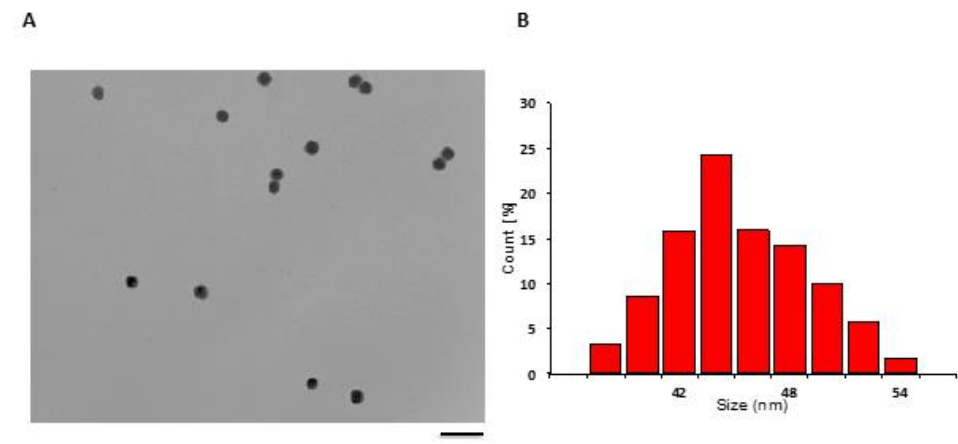

Figure 1. (A) TEM image of $43 \pm 4 \mathrm{~nm}$ spherical AuNPs. (B) Histogram showing the size distribution of spherical AuNPs. Scale bar is $100 \mathrm{~nm}$. 
Particles were found to have a narrow size distribution. The mean size was determined to be $43.5 \mathrm{~nm}$ with a standard deviation of $4.3 \mathrm{~nm}$ (counting 500 NPs using Image $\mathrm{J}$ software). The increase in NP size was also assessed by UVVis spectroscopy as seen in Figure 2.

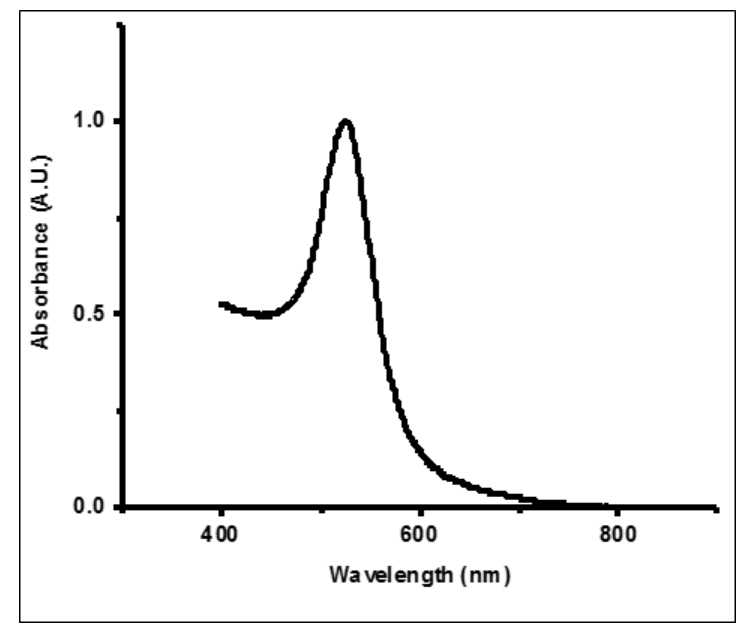

Figure 2. UV-Vis spectra of BSPP coated $43 \pm 4 \mathrm{~nm}$ spherical AuNPs.

Increasing AuNP size is known to cause a red shift in the LSPR band of AuNPs. In this case the plasmon resonance was found to be $528 \mathrm{~nm}$ for $43 \pm 4 \mathrm{~nm}$ BSPP coated AuNPs. This shift, which is in good agreement with previously published results, is attributed to the effect of electromagnetic retardation in larger nanoparticles as well as to contributions from multipolar terms where higher order oscillations start to take important roles. ${ }^{23-26}$ However, an increase in AuNP size also causes a change in the extinction spectrum. This can be explained by an increase in the sphere volume as well as an increase in the relative contribution of scattering to the total extinction $\left(\mathrm{C}_{\mathrm{sca}} / \mathrm{C}_{\mathrm{abs}}\right)$ whereas for smaller size spheres $(<20 \mathrm{~nm})$ the total extinction is nearly all contributed by absorption. These trends therefore suggest that larger NPs $(>40 \mathrm{~nm})$ could be suited for imaging applications. ${ }^{23}$

\subsection{Surface functionalization of spherical AuNPs with synthetic oligonucleotides}

For the functionalization of larger spherical AuNPs $(>40 \mathrm{~nm})$ salt-ageing protocols have been reported. ${ }^{27,} 28$ Nevertheless, it has been found that the oligonucleotide density decreases as the AuNP size increases. This is thought to be due to the surface curvature of spherical AuNPs, which significantly affects the loading of oligonucleotides. Oligonucleotide strands are found closer together thus intensifying inter-strand repulsion. ${ }^{28,29}$ Therefore, the salt-ageing procedure has been stated to not work as efficiently for achieving a high density of oligonucleotides on the surface of larger spherical AuNPs. ${ }^{22,}{ }^{27}$ In order to overcome the limitations associated with the salt-ageing process, a low $\mathrm{pH}$ assisted method was employed for the functionalization of larger spherical AuNPs (see section 2.3 for experimental procedure). This involves the use of a $\mathrm{pH} 3$ citrate buffer, which can lead to efficient DNA adsorption even at low salt concentrations. $^{22}$ The role of the citrate buffer is to overcome the kinetic barrier of DNA attachment, which is more pronounced for larger AuNPs. This occurs via the protonation of bases namely adenine and cytosine at $\mathrm{pH} 3$. These protonated bases reduce the repulsion between the oligonucleotides and AuNPs but most importantly between oligonucleotide strands on a AuNPs surface. ${ }^{22,} 27$ In this study we employed the $\mathrm{pH}$ method for the surface functionalization of $43 \mathrm{~nm}$ spherical AuNPs and UV-Vis spectroscopy was employed to qualitatively assess the oligonucleotide attachment to the NP surface. 

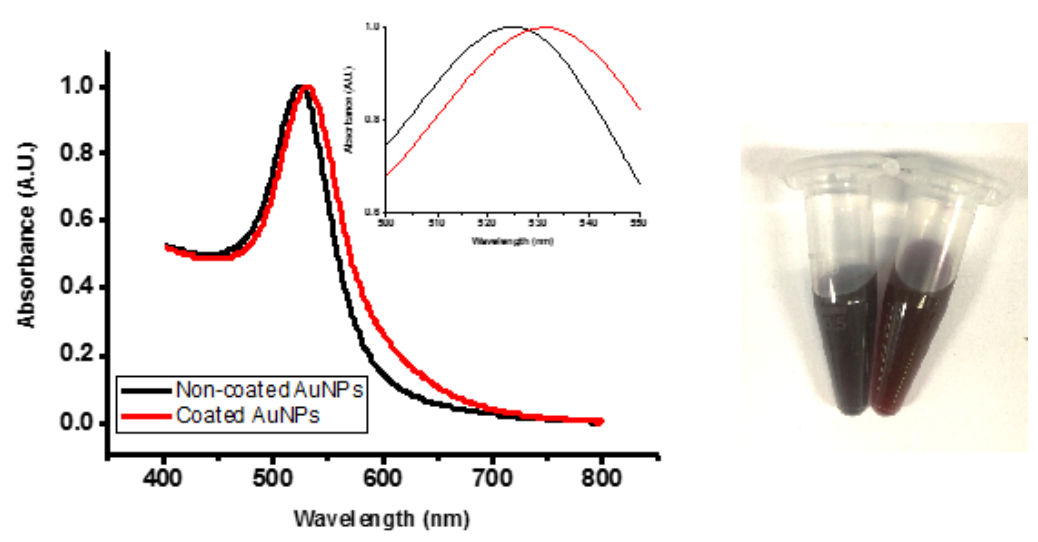

Figure 3. (A) UV-Vis spectra of oligonucleotide coated and non-coated $43 \pm 4 \mathrm{~nm}$ spherical AuNPs. (B) Digital image of non-coated (left Eppendorf) and oligonucleotide coated (right Eppendorf) AuNPs in PBS.

By examining the UV-Vis spectra of both coated and citrate stabilized AuNPs, a red shift of $4 \mathrm{~nm}$ in the SPR peak was observed (see Figure $3 \mathrm{~A}$ ). The absence of a second peak at a longer wavelength also indicates that no aggregation took place during the attachment procedure. ${ }^{30}$ Successful oligonucleotide coating was also assessed by dispersing particles in PBS and comparing it against citrate stabilized spherical AuNPs (see Figure 3 B). Whereas the citrate stabilized nanoparticle solution immediately changed color to dark blue indicating AuNP aggregation due to weak citrate electrostatic stabilization, the oligonucleotide modified spherical AuNPs retained their dark red color.

\section{$3.343 \pm 4$ nm DNA - coated spherical AuNPs for mRNA detection}

$43 \pm 4 \mathrm{~nm}$ AuNPs were coated with a shell of sensing oligonucleotides comprised of a polyT sequence (see section 2.3 for protocol of DNA attachment) designed to detect all mature cellular mRNA via their distinct polyA tail (see Table 1 for detailed oligonucleotide sequence).

Table 1. Sense and flare oligonucleotide sequences. X: thiol modifier 6 S-S (CPG resin from Glen Research)

\begin{tabular}{|c|l|}
\hline Name & Oligonucleotide sequences (5' to 3') and modifications \\
\hline gmRNA (sense strand) & X-AAA CGG GCT TTT TTT TTT TTT TTT TTT TTT TTT TTT T - FAM \\
gmRNA (flare strand) & AAA AAA AAA AAA GCC C - Cy5 \\
\hline
\end{tabular}

DNA - coated AuNPs were further incubated with MRC 5 cells and fixed 18 h post incubation (see section 2.4 and 2.5 for experimental details) in order to preserve and stabilize the cell morphology and allow for continuous imaging over a prolonged period of time without the need of special imaging conditions (temperature of $37{ }^{\circ} \mathrm{C}$ and continuous supply of $\mathrm{CO}_{2}$ ). Prior to TPPL imaging, the sample was imaged via confocal microscopy to ensure that a fluorescence signal due to mRNA sensing could be detectable. A representative confocal image is shown below in Figure 4. 


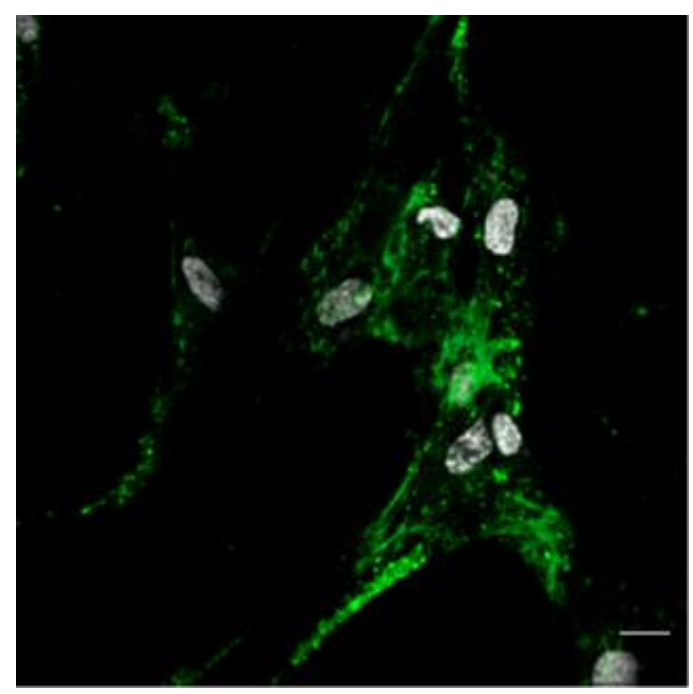

Figure 4. Confocal microscopy image of fixed MRC 5 cells incubated with $43 \pm 4$ nm DNA - coated AuNPs designed to detect all mature cellular mRNA (gmRNA). Colour guide: green - flare release, blue - FAM (dye on sense strand), white nuclear counterstain. Scale bar is $15 \mu \mathrm{m}$.

As can be seen in Figure 4 a fluorescence signal corresponding to detection of all cellular mRNA was detected (green color). The absence of a fluorescence signal from the dye modified sense strand suggested that detection was specific and not due to degradation of surface bound oligonucleotides. After verifying the suitability of the sample, this was further imaged on the setup designed for TPPL and fluorescence imaging.

\subsection{Microscopy setup}

TPPL and fluorescence spectra were acquired on an improved home built setup first presented in Figure 5. The light source for TPPL was a fianium laser $(10 \mathrm{ps}, 20 \mathrm{MHz})$ at a wavelength of $1060 \mathrm{~nm}$ whereas for fluorescence measurements a laser source of $532 \mathrm{~nm}$ or $405 \mathrm{~nm}$ were used for Cy3 (release of flare) and DAPI (nuclear counterstain to image cell nucleus) respectively. The laser beam was guided through a set of mirrors and lenses to a $60 \times$ objective lens. Emission signal was guided back down through the objective and finally filtered by a $430 \mathrm{~nm}$ long pass (LP) and $500 \mathrm{~nm}$ short pass (SP) filter (DAPI) or a $550 \mathrm{~nm}$ LP filter (Cy3) or a $800 \mathrm{~nm}$ SP (TTPL). Depending on the type of measurement the filter was changed accordingly. For clarity a schematic diagram of the setup showing how the laser beam was guided from the laser source, to the sample and back to the detector is shown below in Figure 5. 


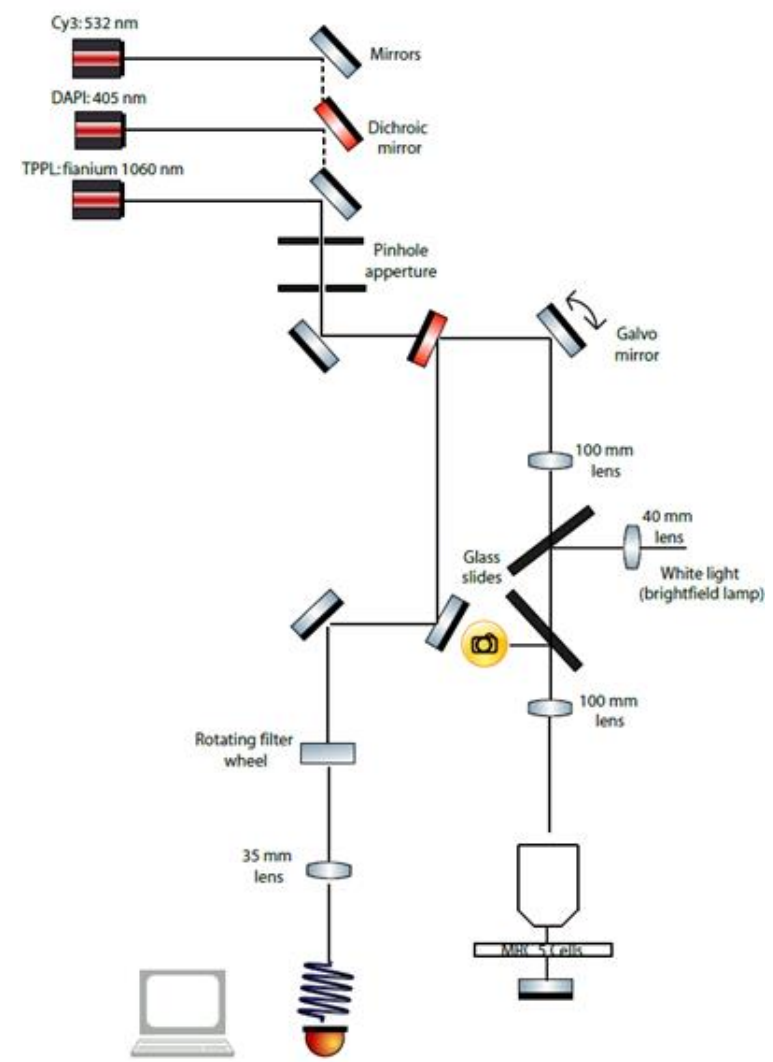

Figure 5. Schematic illustration of the microscopy setup used for both TPPL and fluorescence imaging using the MRC 5 cell line.

The setup was used to further image TPPL and fluorescence corresponding to both Cy3 and DAPI sequentially by using the appropriate laser source and adding the correct filter to the rotating filter wheel as indicated in Figure 6. Using the correct imaging settings the following spectra were acquired for DAPI, Cy3 and TPPL.

DAPI

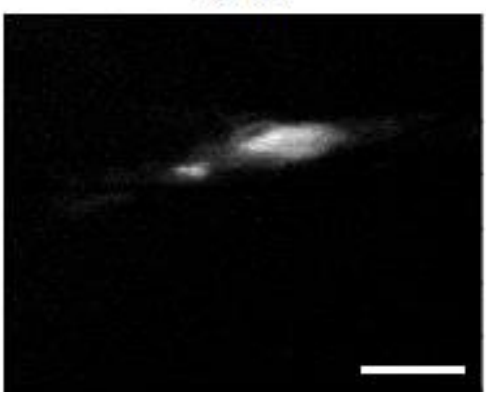

$\mathrm{Cy} 3$

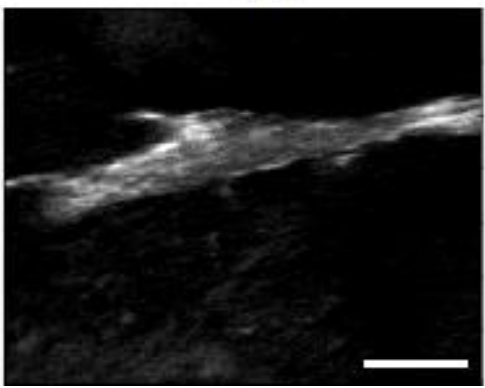

TPPL

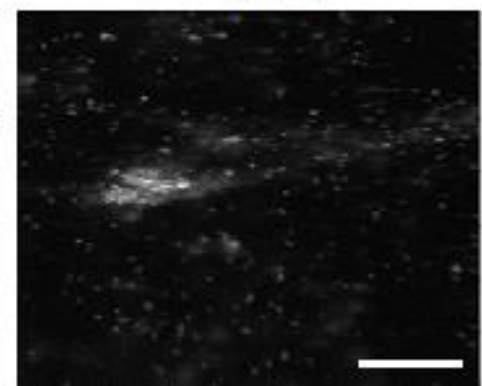

Figure 6. Acquired images showing fluorescence from the cell nucleus (DAPI) and from flare release (Cy3) as well as TPPL from the AuNPs. Scale bars are $15 \mu \mathrm{m}$.

As can be seen from Figure 6 fluorescence from the cell nucleus corresponding to a DAPI signal was clearly imaged. The fluorescence signal showed the characteristic spherical shape of the cell nucleus, which corresponds well with the nuclear shape also imaged on the confocal (Figure 4). This is a strong indication that imaging of the fluorescence signal corresponding to DAPI was successful. Moreover, the Cy3 fluorescence appeared to be located throughout the cell with a stronger intensity being observed at certain sites rather than others. On the other hand a uniform TPPL signal was not imaged throughout the cell with a strong TPPL signal imaged at one position within the cell, which could correlate to 
clusters of nanoparticles located within multivesicular bodies or endosomes (either early or late). However, weaker TPPL signals that may correspond to individual nanoparticles were also imaged. These were located both within the cell as well as extracellularly perhaps from AuNPs that have been exocytosed or were about to be taken up. As the Cy3 signal was diffused throughout the cell upon mRNA detection, co-localisation studies were not possible. For clarity, images presented in Figure 6 were also overlapped (by taking images from one z plane) as shown below in Figure 7.

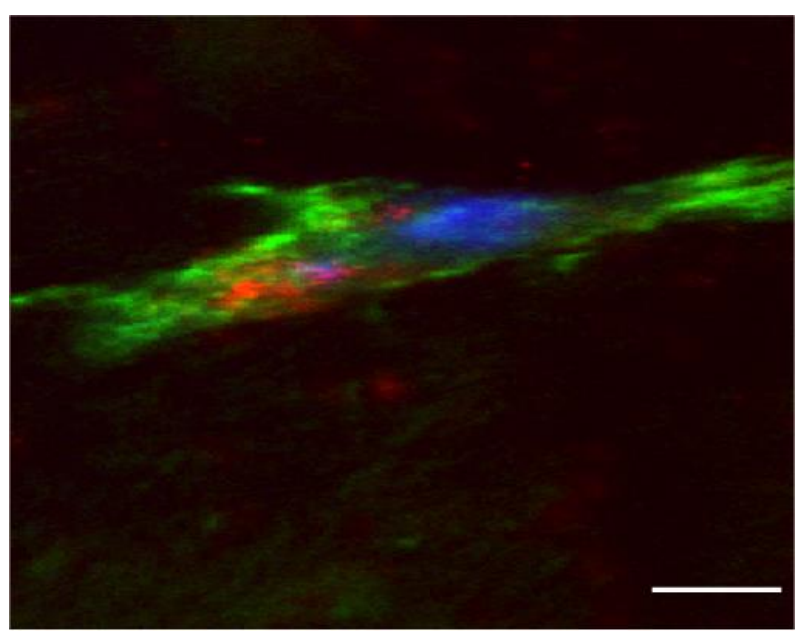

Figure 7. Overlayed images DAPI and Cy3 fluorescence as well as TPPL from the AuNP core. A clear diffuse Cy3 signal was imaged throughout the cell (green), which at points does not co-localize with any TPPL signal (red) and is indicative of cytoplasmic dye diffusion. On the other hand, bright TPPL signals were imaged, which could be due to endosomal localisation. Furthermore, weak TPPL signals were imaged extracellularly, which could be due to NP exocytosis as imaging in the perimeter of another cell as Cy3 fluorescence could also be observed at the bottom of the image. Scale bar is $15 \mu \mathrm{m}$.

Apart from imaging in the $x$ and $y$ direction creating a 2D image, as seen in Figure 7, an image at the same position was also acquired in the $\mathrm{z}$ direction creating 3D images of DAPI and Cy3 fluorescence as well as TPPL as seen in Figure 8.

DAPI
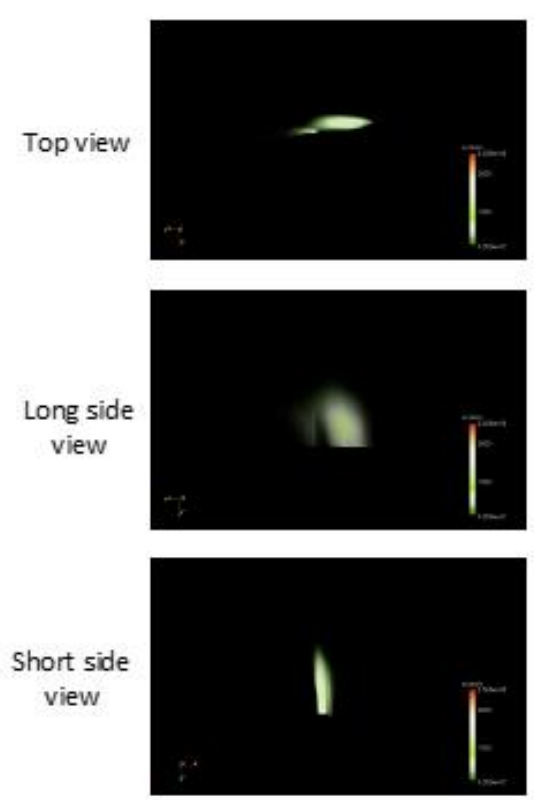

Cy3
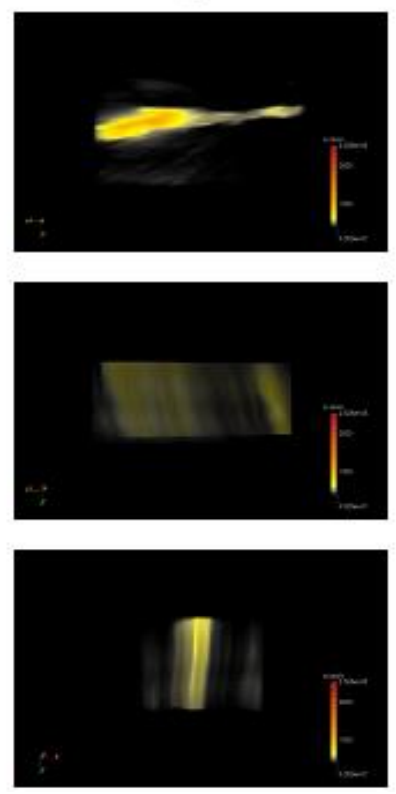

TPPL
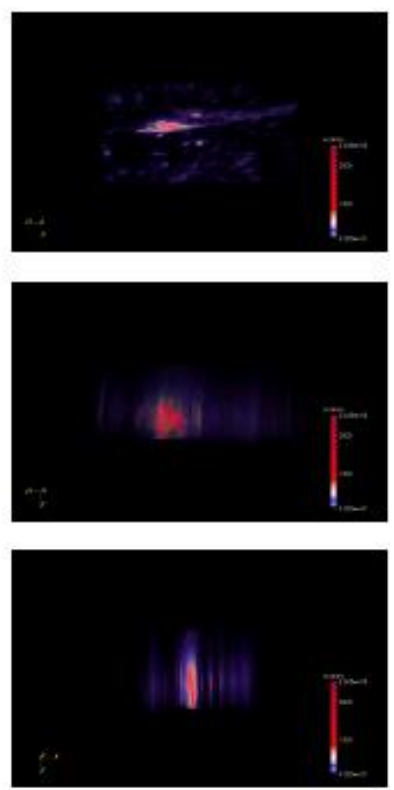

Figure 8. 3D images created by imaging in the $\mathrm{z}$ direction for DAPI and Cy3 fluorescence as well as TPPL from the AuNP core. Images were analyzed and presented as a top view, long side and short side view for each scan. 
Once more it can be seen that although the nucleus is well defined, the fluorescence signal corresponding to flare release is diffused and observed throughout the cell. On the other hand, a TPPL signal when analyzed in each direction did not appear to be well-dispersed throughout the cell which is a strong indication that flare release is imaged within the cytoplasm leading to an almost uniform fluorescence signal whereas the AuNPs are most likely located within endosomes leading to a very bright aggregated TPPL signal with a weaker signal being imaged in areas, which may correspond to single particles located within the cytoplasm following endosomal escape.

\section{CONCLUSION}

In conclusion, by using DNA-coated $43 \pm 4 \mathrm{~nm}$ AuNPs we demonstrated how a home-built microscopy set-up could be developed capable of imaging both the gold core via TPPL and the fluorescence from the release of the flare within fixed MRC 5 cells. Future experiments will include the endocellular TPPL imaging of nanoparticles of different shapes.

\section{REFERENCES}

[1] Cutler, J. I.; Auyeung, E.; Mirkin, C. A., Spherical Nucleic Acids. J. Am. Chem. Soc. 2012, 134 (3), $1376-1391$.

[2] Heuer-Jungemann, A.; Harimech, P. K.; Brown, T.; Kanaras, A. G., Gold nanoparticles and fluorescentlylabelled DNA as a platform for biological sensing. Nanoscale 2013, 5 (20), 9503-9510.

[3] Seferos, D. S.; Giljohann, D. A.; Hill, H. D.; Prigodich, A. E.; Mirkin, C. A., Nano-flares: Probes for transfection and mRNA detection in living cells. J. Am. Chem. Soc. 2007, 129 (50), 15477-+.

[4] Prigodich, A. E.; Seferos, D. S.; Massich, M. D.; Giljohann, D. A.; Lane, B. C.; Mirkin, C. A., Nano-flares for mRNA Regulation and Detection. Acs Nano 2009, 3 (8), 2147-2152.

[5] Gao, N. Y.; Chen, Y.; Li, L.; Guan, Z. P.; Zhao, T. T.; Zhou, N.; Yuan, P. Y.; Yao, S. Q.; Xu, Q. H., ShapeDependent Two-Photon Photoluminescence of Single Gold Nanoparticles. Journal of Physical Chemistry C 2014, 118 (25), 13904-13911.

[6] Kyriazi, M. E.; Giust, D.; El-Sagheer, A. H.; Lackie, P. M.; Muskens, O. L.; Brown, T.; Kanaras, A. G., Multiplexed mRNA Sensing and Combinatorial-Targeted Drug Delivery Using DNA-Gold Nanoparticle Dimers. Acs Nano 2018, 12 (4), 3333-3340.

[7] Heuer-Jungemann, A.; El-Sagheer, A. H.; Lackie, P. M.; Brown, T.; Kanaras, A. G., Selective killing of cells triggered by their mRNA signature in the presence of smart nanoparticles. Nanoscale 2016, 8 (38), 16857-16861.

[8] Vilela, P.; Heuer-Jungemann, A.; El-Sagheer, A.; Brown, T.; Muskens, O. L.; Smyth, N. R.; Kanaras, A. G., Sensing of Vimentin mRNA in 2D and 3D Models of Wounded Skin Using DNA-Coated Gold Nanoparticles. Small 2018, 14 (12).

[9] McClellan, S.; Slamecka, J.; Howze, P.; Thompson, L.; Finan, M.; Rocconi, R.; Owen, L., mRNA detection in living cells: A next generation cancer stem cell identification technique. Methods 2015, 82, 47-54.

[10] Seftor, E. A.; Seftor, R. E. B.; Weldon, D. S.; Kirsammer, G. T.; Margaryan, N. V.; Gilgur, A.; Hendrix, M. J. C., Melanoma Tumor Cell Heterogeneity: A Molecular Approach to Study Subpopulations Expressing the Embryonic Morphogen Nodal. Semin. Onc. 2014, 41 (2), 259-266.

[11] Li, B. J.; Menzel, U.; Loebel, C.; Schmal, H.; Alini, M.; Stoddart, M. J., Monitoring live human mesenchymal stromal cell differentiation and subsequent selection using fluorescent RNA-based probes (vol 6, 26014, 2016). Sci. Rep. 2016, 6 .

[12] Tu, Y. Q.; Wu, P.; Zhang, H.; Cai, C. X., Fluorescence quenching of gold nanoparticles integrating with a conformation-switched hairpin oligonucleotide probe for microRNA detection. Chemical Communications 2012, 48 (87), 10718-10720.

[13] J., L.; J., H.; X., Y.; Y., Y.; K., Q.; N., X.; C., M.; K., W., Two-Color-Based Nanoflares for Multiplexed MicroRNAs Imaging in Live Cells. Nanotheranostics 2018, 2 (1), 96-105.

[14] Wu, X. A.; Choi, C. H. J.; Zhang, C.; Hao, L.; Mirkin, C. A., Intracellular Fate of Spherical Nucleic Acid Nanoparticle Conjugates. J. Am. Chem. Soc. 2014, 136 (21), 7726-7733. 
[15] Durr, N. J.; Larson, T.; Smith, D. K.; Korgel, B. A.; Sokolov, K.; Ben-Yakar, A., Two-photon luminescence imaging of cancer cells using molecularly targeted gold nanorods. Nano Letters 2007, 7 (4), 941-945.

[16] van den Broek, B.; Ashcroft, B.; Oosterkamp, T. H.; van Noort, J., Parallel Nanometric 3D Tracking of Intracellular Gold Nanorods Using Multifocal Two-Photon Microscopy. Nano Letters 2013, 13 (3), 980-986.

[17] Tong, L.; Wei, Q. S.; Wei, A.; Cheng, J. X., Gold Nanorods as Contrast Agents for Biological Imaging: Optical Properties, Surface Conjugation and Photothermal Effects. Photochemistry and Photobiology 2009, 85 (1), 21-32.

[18] Wang, H. F.; Huff, T. B.; Zweifel, D. A.; He, W.; Low, P. S.; Wei, A.; Cheng, J. X., In vitro and in vivo twophoton luminescence imaging of single gold nanorods. Proceedings of the National Academy of Sciences of the United States of America 2005, 102 (44), 15752-15756.

[19] Liu, Y.; Ashton, J. R.; Moding, E. J.; Yuan, H. K.; Register, J. K.; Fales, A. M.; Choi, J.; Whitley, M. J.; Zhao, X. G.; Qi, Y.; Ma, Y.; Vaidyanathan, G.; Zalutsky, M. R.; Kirsch, D. G.; Badea, C. T.; Vo-Dinh, T., A Plasmonic Gold Nanostar Theranostic Probe for In Vivo Tumor Imaging and Photothermal Therapy. Theranostics 2015, 5 (9), $946-960$. [20] Rane, T. D.; Armani, A. M., Two-Photon Microscopy Analysis of Gold Nanoparticle Uptake in 3D Cell Spheroids. Plos One 2016, 11 (12).

[21] Bastus, N. G.; Comenge, J.; Puntes, V., Kinetically Controlled Seeded Growth Synthesis of Citrate-Stabilized Gold Nanoparticles of up to 200 nm: Size Focusing versus Ostwald Ripening. Langmuir 2011, 27 (17), 11098-11105.

[22] Zhang, X.; Servos, M. R.; Liu, J., Instantaneous and Quantitative Functionalization of Gold Nanoparticles with Thiolated DNA Using a pH-Assisted and Surfactant-Free Route. Journal of the American Chemical Society 2012, 134 (17), 7266-7269.

[23] Jain, P. K.; Lee, K. S.; El-Sayed, I. H.; El-Sayed, M. A., Calculated absorption and scattering properties of gold nanoparticles of different size, shape, and composition: Applications in biological imaging and biomedicine. Journal of Physical Chemistry B 2006, 110 (14), 7238-7248.

[24] Berciaud, S.; Cognet, L.; Tamarat, P.; Lounis, B., Observation of intrinsic size effects in the optical response of individual gold nanoparticles. Nano Letters 2005, 5 (3), 515-518.

[25] El-Sayed, M.; Huang, X., Gold nanoparticles: Optical properties and implementations in cancer diagnosis and photothermal therapy. Journal of Advanced Research 2010, 1 (1), 13-28.

[26] Link, S.; El-Sayed, M. A., Size and temperature dependence of the plasmon absorption of colloidal gold nanoparticles. Journal of Physical Chemistry B 1999, 103 (21), 4212-4217.

[27] Liu, B.; Liu, J. W., Methods for preparing DNA-functionalized gold nanoparticles, a key reagent of bioanalytical chemistry. Analytical Methods 2017, 9 (18), 2633-2643.

[28] Hurst, S. J.; Lytton-Jean, A. K. R.; Mirkin, C. A., Maximizing DNA loading on a range of gold nanoparticle sizes. Anal. Chem. 2006, 78 (24), 8313-8318.

[29] Hill, H. D.; Millstone, J. E.; Banholzer, M. J.; Mirkin, C. A., The Role Radius of Curvature Plays in Thiolated Oligonucleotide Loading on Gold Nanoparticles. Acs Nano 2009, 3 (2), 418-424.

[30] Zhang, Z.; Wang, L.; Wang, J.; Jiang, X.; Li, X.; Hu, Z.; Ji, Y.; Wu, X.; Chen, C., Mesoporous Silica-Coated Gold Nanorods as a Light-Mediated Multifunctional Theranostic Platform for Cancer Treatment. Advanced Materials 2012, 24 (11), 1418-1423. 